\section{Response to: 'The effect of synovial tissue volume shrinking on pain relief for knee osteoarthritis was overestimated or not?' by Wei et al}

We thank Wei et al for their interest in our work and positive comments about our trial results. ${ }^{1}{ }^{2}$ Wei et al highlight a number of issues that we address in turn. Analysis of data from our trial of patients with symptomatic knee osteoarthritis (OA) showed an association between change in the level of knee pain and change in synovial tissue volume (STV) following an intra-articular steroid injection. We agree with Wei et al that synovial shrinkage is not the only mechanism by which steroids might result in a reduction in pain; this is underscored, as we highlight in our paper, by the fact that STV accounted for only a small proportion of the variance in change in pain. We think it is unlikely though that our observed findings overestimate the relationship between STV shrinkage and change in pain. As noted by Wei et al, only $6.4 \%$ of participants had a KellgrenLawrence $(\mathrm{K} / \mathrm{L})$ radiographic grade 4 . In sensitivity analyses in which we removed those, our results were unchanged and remained highly significant. Wei et al ask about our approach to analysis of the data, which featured longitudinal data over three study visits. A straightforward linear regression or pairwise correlation (such as Spearman's or Pearson's) would not sufficiently account for the repeated measures on the same patient (and the fact that each patient's observations are therefore correlated), and therefore, the SEs calculated for the model would be unnecessarily large. We also had more than two visits, and so a simple change-versus-change (change in pain vs change in STV) correlation would also mean losing data from the third visit, reducing our sample size unnecessarily. A fixed-effects linear regression allowed us to compare pain and STV using data from all three visits, while removing the effect of change over time in the appropriate manner, accounting for within-patient correlation, and adjusting the SEs in the correct way, which is why we opted for this methodology. More details on the use of these models are given in Gardiner et al. ${ }^{3}$ In our trial, we included subjects with symptomatic knee OA irrespective of OA compartment subtype and did make any adjustments for this in the analysis. In addition to overall $\mathrm{K} / \mathrm{L}$ grade, there was no difference in tibiofemoral $\mathrm{K} / \mathrm{L}$ grade between responders and non-responders. We did not track dosage change of analgesia; generally, however, medication use parallels pain change (ie, pain reduction and rescue medication reduction tend to go together). Lastly, we feel that the term 'within $20 \%$ ' is preferable to the term 'over 20\%' in defining response because it refers to the patient's pain worsening, following an initial response, to a score of 'within' $20 \%$ above the baseline value.

Terence W O'Neill, ${ }^{1,2,3}$ Matthew J Parkes, ${ }^{1,2}$ Nasimah Maricar, ${ }^{1,2}$ Elizabeth J Marjanovic, ${ }^{1,2}$ Richard Hodgson, ${ }^{4}$ Andrew D Gait, ${ }^{4}$ Timothy F Cootes, ${ }^{4}$ Charles E Hutchinson, ${ }^{5}$ David T Felson ${ }^{1,2,6}$

${ }^{1}$ Faculty of Medical and Human Sciences, Arthritis Research UK Centre for Epidemiology, Institute of Inflammation and Repair, Manchester Academic Health Science Centre, University of Manchester, Manchester, UK

${ }^{2}$ NIHR Manchester Musculoskeletal Biomedical Research Unit, Central Manchester NHS Foundation Trust, Manchester Academic Health Science Centre, Manchester, UK

${ }^{3}$ Department of Rheumatology, Salford Royal NHS Foundation Trust, Salford, UK

${ }^{4}$ Centre of Imaging Sciences, Institute of Population Health, University of Manchester, Manchester, UK

${ }^{5}$ UK Warwick Medical School, The University of Warwick, Coventry, UK

${ }^{6}$ Clinical Epidemiology Unit, Boston University School of Medicine, Boston, Massachusetts, USA

Correspondence to Dr Terence W O'Neill, Arthritis Research UK Centre for Epidemiology, University of Manchester, Manchester M13 9PT, UK; terence.o'neill@manchester.ac.uk

Twitter Follow Matthew Parkes at @mattyjparkes

Contributors TWO, MJP and DTF drafted the response. NM, EJM, RH, ADG, TFC and CEH reviewed the reply.

Funding Arthritis Research UK (grant no. 18676).

Competing interests None declared.

Ethics approval Leicestershire Multicentre Research Ethics Committee.

Provenance and peer review Commissioned; internally peer reviewed.

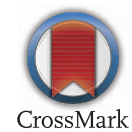

To cite O'Neill TW, Parkes MJ, Maricar N, et al. Ann Rheum Dis 2015;74:e65.

Received 31 July 2015

Accepted 3 August 2015

Published Online First 7 September 2015

\section{SLinked}

http://dx.doi.org/10.1136/annrheumdis-2015-208223

Ann Rheum Dis 2015;74:e65. doi:10.1136/annrheumdis-2015-208233

\section{REFERENCES}

1 Wei J, Zeng C, Lei GH. The effect of synovial tissue volume shrinking on pain relief for knee osteoarthritis was overestimated or not? Ann Rheum Dis 2015;74:e64.

2 O'Neill TW, Parkes MJ, Maricar N, et al. Synovial tissue volume: a treatment target in knee osteoarthritis (OA). Ann Rheum Dis 2015. Published Online First: 26 Jun 2015. doi:10.1136/annrheumdis-2014-206927

3 Gardiner JC, Luo Z, Roman LA. Fixed effects, random effects and GEE: what are the differences? Stat Med 2009:28:221-39. 